\title{
GÉNERO COMO FACTOR QUE INCIDE EN ASESORÍAS EN CÁLCULO
}

\section{GENDER AS A FACTOR THAT AFFECTS CONSULTANCY IN CALCULUS}

\author{
Martha Cecilia Santiago-Carrillo ${ }^{1}$ \\ Eduardo lbarguen Mondragón ${ }^{2}$ \\ Mawency Vergel-Ortega ${ }^{3}$
}

\section{RESUMEN}

La investigación desde un enfoque cuantitativo analiza los factores y el impacto de la unidad de servicios académicos intensivos en los resultados del aprendizaje en matemáticas y física aplicadas, teniendo en cuenta la perspectiva de género en las instituciones de educación básica y de educación secundaria profesional oficial de la ciudad de CúcutaColombia. Se incorporan como instrumentos los registros del proyecto pedagógico de la Clínica de Matemáticas y la prueba de aptitud-actitud en Cálculo aplicado a la física. Los instrumentos registran una alta confiabilidad con un índice kappa superior a 0,7, y un índice alfa de 0,78 en

1 Departamento de Matemáticas y Estadística, Universidad Francisco de Paula Santander, Norte de Santander, Cúcuta, Colombia. E-mail: marcesa2010@gmail.com https://orcid.org/0000-0002-2124-7438

2 Departamento de Matemáticas y Estadística, Universidad de Nariño, Pasto, Colombia. E-mail: edbargun@ edunar.edu.co https://orcid.org/0000-0001-6308-1344

3 Departamento de Matemáticas y Estadística, Universidad Francisco de Paula Santander, Norte de Santander, Cúcuta, Colombia. E-mail: mawencyvergel@ufps.edu.co https://orcid.org/0000-0001-8285-2968 la prueba. Los resultados muestran factores asociados a las diferencias de género en cuanto a motivación, comportamiento y razonamiento mecánico; se concluye con un $95 \%$ de confianza que existen diferencias de género significativas en la formación científica en física, donde los estudiantes varones tienen más probabilidades de asistir a los servicios de asesoría debido a la mayor dificultad para resolver problemas en física y matemáticas aplicadas; las estudiantes mujeres muestran mayor consistencia en la participación y actividad creativa en su formación en cálculo

PALABRAS CLAVE: Género, clínica matemática, formación en ciencia.

\section{ABSTRACT}

The research from a quantitative approach analyzes the factors and impact of the intensive academic services unit on learning outcomes in applied mathematics and physics, taking into account the gender perspective in the 
institutions of basic education and official secondary professional education in the city of Cúcuta-Colombia. The records of the pedagogical project of the Mathematics Clinic and the aptitude-attitude test in calculus and physics are incorporated as instruments. The instruments register a high reliability with a kappa index of 0.79 and 0.8 respectively, and an alpha index of 0.78 in the test. The results show factors associated with gender differences in motivation, behavior, and mechanical reasoning; it is concluded with $95 \%$ confidence that significant gender differences exist in scientific training in physics, where male students are more likely to attend counseling services due to greater difficulty in solving problems in physics and applied mathematics; female students show greater consistency in participation and creative activity in their training in calculus and physics.

KEYWORDS: Gender, physics, clinical math, science education.

\section{INTRODUCCIÓN}

La formación en cálculo aplicado ha realizado profundas transformaciones, desde la educación infantil hasta la educación superior [1], realizando cambios en el estilo tradicional, donde las investigaciones indican que en esta área, el profesor era considerado como una figura sin empatía hacia el estudiante, el único dueño del conocimiento e intransmisible [2], donde sólo se comparten fórmulas, donde el joven no potencia su pensamiento creativo considerando intrascendente esta ciencia; problemas que conducen a la deserción [3], a bajos niveles de desarrollo del pensamiento científico y creativo, sumado a ello, el estudiante pierde el interés por el aprendizaje y le lleva a un bajo rendimiento académico, a la pérdida de comunicación entre las ciencias. La Clínica de Matemáticas ha sido una estrategia que busca mejorar y dar soluciones a estos problemas[2], para que se apoye el trabajo y la imagen del profesor, donde deja de ser un transmisor de conocimientos en física y toma conciencia de que su función es generar ambientes y herramientas con posibilidades para que los estudiantes produzcan y construyan conocimientos y hagan innovaciones en física, que sientan placer y satisfacción en hacer diseños validados por esta ciencia, en descubrir y no sólo reproducir, utilizando el método científico. La orientación en física y matemáticas aplicadas, tiene como deber urgente preparar a los niños y jóvenes para resolver los problemas de la vida y esto se logra no sólo con la transmisión de conocimientos, sino potenciando, asesorando, generando herramientas, métodos y estrategias de aprendizaje que permitan la búsqueda del conocimiento, donde la ciencia pueda ser aplicada y generada. Asimismo, los padres de familia en las instituciones observan problemas asociados con el género y la discriminación de la mujer en el aprendizaje de esta ciencia, no teniendo las mismas oportunidades, ni permitiéndoles hacer aportes en las clases, ni ser líderes de equipos en el aula, situaciones.

Los estudios de género en diferentes ámbitos educativos, sociales, económicos y culturales, según [4] han trascendido los límites biológicos y sexuales para interesarse por su carga simbólica, es decir, por la forma en que las personas se relacionan y se presentan ante la sociedad desde su experiencia al identificarse como de un género u otro. En la perspectiva [5] se ha estudiado la incidencia del género en el rendimiento matemático durante 40 años, algunos autores representativos [6] encontraron que los niños se desempeñan mejor con los números y las niñas con las humanidades, sin embargo, estudios posteriores no han tenido resultados concluyentes. Posteriormente, la teoría feminista consideró a las matemáticas como un filtro crítico para incentivar la participación de las mujeres en el mercado laboral como ingenieras, científicas o programadoras [7], a tal punto que, por su relevancia e impacto social, la diferencia en el 
acercamiento de los niños y adolescentes (NNA) al conocimiento numérico [8], se constituye como una motivación para desarrollar el tema en este artículo.

El propósito de los estudios centrados en el género dentro del campo de las matemáticas es fortalecer el rendimiento de todos los estudiantes, minimizando los obstáculos socioculturales [9] que se derivan de décadas de condicionamientos y limitaciones [10], uno de los autores de referencia en este campo [11] para el que el individuo es ante todo un ser humano, constructor de conocimiento, dentro de su entorno sociocultural, por lo que la relación que establezca con las matemáticas estará en función de su entorno social y de las experiencias que estén impregnadas del sexo al que pertenezca, dadas las atribuciones sociales que se le han dado a cada uno (hombre, mujer), esto desde una perspectiva de investigación socio-epistemológica.

En la misma línea, [12] ha señalado que las diferencias de género en el rendimiento matemático implican diferentes aspectos, entre ellos la genética, pero también los estereotipos culturales que afectan a los profesores, los padres y las comunidades, así como el acceso a las oportunidades, ya que la realidad socioeconómica de todos los estudiantes es diferente y cuando se encuentran en un estado de necesidad o vulnerabilidad son las niñas las que, según el autor, se enfrentan a las mayores dificultades. Por su parte, [13] han destacado la oportunidad de incorporar los principios de la psicología social para promover la motivación como herramienta para minimizar el impacto de los estereotipos culturales. En los estudios [14], la participación de niños y niñas en programas o concursos especiales de matemáticas durante la infancia está distribuida de manera uniforme, pero luego en la adolescencia disminuye drásticamente, ya que los niños reportan alrededor del $73 \%$ frente al $27 \%$ de las niñas.
En el contexto de la Clínica de Matemáticas, el impacto no sólo ha acercado a los estudiantes a la asignatura, sino que también ha generado un espacio amigable e inclusivo para todos ellos, sin marcar ningún tipo de prejuicio o diferenciación. En este sentido [15] consideran fundamental desarrollar estetipo desituaciones de aprendizaje desde la nueva perspectiva socio-epistemológica en la que la atención de los niños es atraída por medio de un desafío y así llevarlos a poner en práctica todas las herramientas matemáticas que han desarrollado hasta el momento. Según [16] el talento para las matemáticas y la física no es una construcción fija sino desarrollable. En este sentido, es posible diseñar eficazmente entornos y dinámicas de aprendizaje en los que los procesos, las interacciones y los valores pueden tener en cuenta los aspectos históricos o culturales que pretenden clasificar y limitar a los niños y adolescentes.

La posibilidad de incluir el género en las estrategias es un camino que cada vez tiene más adeptos, una decisión social y pedagógica conectada con la actualidad. Es por ello que los investigadores diseñaron como estrategia anexa a las matemáticas clínicas, la Unidad de Servicios Académicos Inmediatos hacia la formación de científicos USI, acogiendo los problemas de física desde el asesoramiento de las matemáticas aplicadas, unidad en la que se recrean escenarios de asistencia en los experimentos, asesoramiento en la modelización, gestión de recursos en tecnologías de la comunicación y la información para grandes datos, y planteando como hipótesis, la USI puede potenciar la formación en pensamiento creativo científico en física y matemáticas aplicadas, ¿Qué factores se asocian con la diferencia de género en los procesos de enseñanza de la física? En cuanto a la diferencia de género, ¿cuál es la actitud hacia la USI? 


\section{METODOLOGÍA}

El enfoque de la investigación es cuantitativo, correlativo y causal [17]. En conjunto, esta elección permite inferir de la muestra la incidencia de género, la participación, el interés y el compromiso de los alumnos de quinto grado de primaria, así como en el sexto grado de básica secundaria, y en el décimo y el undécimo grado de la educación media, lo que redunda en el interés de formular algunas reflexiones, que pueden beneficiar a otros centros educativos con iniciativas pedagógicas similares. Asimismo, se considera que se siguen los principios de los estudios descriptivos en los que se pueden detallar situaciones 0 acontecimientos, profundizando en cómo es y cómo se manifiesta un determinado fenómeno, a partir de la identificación de las propiedades y características más relevantes o esenciales de las personas y grupos analizados [18].

El proyecto de la Clínica de Matemáticas está en funcionamiento y se ha convertido en un proyecto educativo que ofrece apoyo a los estudiantes de primaria, secundaria y educación media que tienen dificultades en el rendimiento matemático. La estructura de la Clínica sigue los lineamientos generales de un centro médico, en ese sentido cuenta con un área de Emergencias donde se reciben a los estudiantes con dificultades de aprendizaje que desean fortalecer su desempeño y también cuenta con una Unidad Académica de Servicios Inmediatos (USI) donde los estudiantes son remitidos por el profesor a cargo de la materia y necesitan recuperar los logros perdidos. En este sentido, los docentes y pasantes [1] de la Universidad Francisco de Paula Santander han identificado que la mayor dificultad se ha centrado en las habilidades de resolución de problemas en física o matemáticas aplicadas [2], asimismo, la participación según el género ha representado diferencias significativas en la actitud y el compromiso de los estudiantes.
Durante su operación de asistencia personalizada, han tenido la oportunidad de acompañar la evolución de los estudiantes e identificar algunos patrones de comportamiento que no sólo les sirven en el campo pedagógico, sino que también les permiten tomar mejores decisiones dentro del proceso. Otro estudio [9] destaca la necesidad de evitar los estereotipos y prejuicios en los análisis de rendimiento basados en el género, ya que no son indicadores fiables, sino que pueden sesgar los estudios. En la misma línea [10] afirman que es hora de superar las perspectivas de la Grecia clásica donde el género era un factor para considerar a las personas más o menos inteligentes.

La población objeto comprende 129 jóvenes de 3 instituciones educativas oficiales de la ciudad de Cúcuta-Colombia. Los participantes fueron seleccionados por el método de muestreo no probabilístico por conveniencia, incidental 0 coincidencia, provoca la asistencia a la USI en el asesoramiento sobre cuestiones de física y cálculo aplicado; las edades de la muestra están entre 10 y 18 años $(M=14,93, S D=1,696$, Rango=10-18) y según la variable de género, hay porcentajes bastante equilibrados, $46 \%$ de hombres y $54 \%$ de mujeres. El $60,7 \%$ corresponde al estrato medio-bajo $0-1$ y el $28,3 \%$ al estrato medio 3. En el desarrollo de la investigación se implementan cuestionarios y pruebas de aptitud en física. La investigación se desarrolla a través de fases, estas son: diagnóstico inicial, análisis de datos y evaluación. En la fase de diagnóstico se analizan los informes y registros del proyecto, como los formatos de la historia clínica y los registros de atención.

En el análisis del cuestionario de aptitudactitud, se realizó una primera evaluación para contemplar la existencia de diferencias significativas en la aptitud total en física, utilizando el género como variable independiente, mediante el test ANOVA. Después de evaluar estas diferencias, se continuó el análisis de 
la consistencia interna del cuestionario. Para evaluar la fiabilidad se utilizó el test de Cronbach a y el test de Spearman-Brown de dos mitades con alfa=0,75. A continuación se aplicaron los cálculos para comprobar la validez factorial. En una segunda fase, la de análisis de datos, la información recogida se organiza en gráficos estadísticos que permiten comprender mejor el alcance del proyecto y también consolida los gráficos centrados en el impacto del género en el proceso de enseñanza-aprendizaje desde el punto de vista de la asistencia, la participación y el compromiso, tres criterios clave para el análisis.

En la fase de evaluación, por último, se realiza un análisis discursivo de los resultados, confrontando lo que algunos autores clave han dicho sobre el tema del género y los logros en matemáticas o el género y la educación. En esta medida, los resultados obtenidos se consolidan y se centran en la contribución que representan para el proyecto y para otros posibles lectores interesados en este tipo de experiencia pedagógica.

\section{RESULTADOS}

Losresultados deltestANOVAponendemanifiesto la existencia de diferencias significativas la un nivel $p<0,05)$ en los niveles totales de las instituciones educativas, dependiendo de la variable de género. Se observaron diferencias significativas en la prueba de aptitud física $(p=0,025)$, siendo las mujeres $(-x=125,7)$ las que mostraron resultados superiores en los hombres $\left({ }^{-} x=115,04\right)$. La consistencia interna del cuestionario fue de 0,76 para la muestra masculina y de 0,77 para la femenina. En la prueba de dos mitades de Spearman-Brown, el coeficiente fue de 0,81 para los hombres y 0,812 para las mujeres. Se observa que no hay una diferencia significativa con una fiabilidad del $95 \%$ en ambos géneros, incluyendo todos los ítems. Esto muestra una alta fiabilidad en el cuestionario desarrollado, independientemente del género.

En cuanto a la participación y asistencia a la USI, desde el inicio de la implementación de la Clínica de Matemáticas, se observa que los estudiantes varones son más receptivos al proyecto. Sin embargo, inicialmente la asistencia fue más simétrica, el $55 \%$ de ellos y el $45 \%$ de ellos y los registros mantenidos por el equipo, se evidenció que los niños son menos tímidos a la hora de reconocer que no entienden un tema y pedir ayuda. Las niñas tratan de resolver los ejercicios por su cuenta primero, y sólo cuando descubren que algo está persistentemente mal deciden buscar ayuda. Existe una alta tendencia de participación semanal en la USI con 90 asistentes, en la que el $60 \%$ de los participantes de la clínica en las Instituciones educativas oficiales pertenecen al género masculino y el $40 \%$ al femenino.

Los factores encontrados entre las mujeres fueron cognitivos, motivacionales, de razonamiento, de comportamiento. Para el primer factor, que combina elementos relacionados con el factor conductual, obtuvo un resultado de 0,98; el factor cognitivo, obtuvo un valor de 0,87, el factor de motivación fue de 0,77. El tercer factor, que se refiere a la habilidad en el razonamiento mecánico, obtuvo una fiabilidad de 0,715. La consistencia interna de Cronbach con $a=0,7$. Spearman-Brown para los factores fue de $0,9,0,7,0,6$ y 0,6 respectivamente. Para el cuestionario de aptitud-actitud fue de 0,75. Los factores en el género masculino fueron seis factores, a saber, el autocontrol, obtuvo un valor de 0,9, el razonamiento mecánico 0,81, el cognitivo 0,68, el autoconcepto obtuvo una fiabilidad de 0,65, la motivación fue de 0,61 y el conductual con alfa de 0,6 . Los coeficientes del segundo análisis para los factores fueron 0,79 , $0,76,0,71,0,65$ y 0,6 respectivamente.

La correlación en la prueba implementada a hombres y mujeres de los elementos fue positiva 
indicando que los valores de ambos elementos varían de manera similar. La correlación fue significativa en el nivel $p=0,01$. Las correlaciones con los niveles superiores $r=0,91 p=0,01$, se encuentran en el factor de comportamiento/ razonamiento mecánico para las mujeres y $r=0,92$ para la motivación/razonamiento mecánico. Sólo los factores que coinciden en ambos géneros fueron considerados para ANOVA con los mismos ítems), apareciendo categorías en motivación $p=0$, conductual $p=0$, razonamiento mecánico $p=0$, las diferencias son estadísticamente significativas, mientras que para el factor cognitivo $p=0,61$ no lo fueron. En los casos en que las diferencias fueron significativas, la muestra femenina refleja resultados significativamente más altos en la motivación $\left(x^{-}=1,95\right.$ para las mujeres $y x^{-}=0,87$ para los hombres; en la conductual $x^{-}=1,9$ para los hombres $\mathrm{y} \mathrm{x}^{-}=1,1$ para las mujeres, en el razonamiento mecánico $x^{-}=1,97$ para los hombres y $\mathrm{x}^{-}=1,5$ para las mujeres). Se observa que la variable de género influye en los factores.

\section{CONCLUSIONES}

Según la investigación, se encontró que existen diferencias significativas en torno al aprendizaje del cálculo asociadas al género de los estudiantes, con factores aptitud en la física, la actitud en la física, donde los estudiantes varones tienen más probabilidades de asistir a los servicios de asesoramiento debido a la mayor dificultad para resolver problemas en cálculo aplicado; las estudiantes de identidad mujer muestran una mayor consistencia en la participación y la actividad creativa en su formación.

Los factores relacionados con las instituciones educativas oficiales son la motivación, el comportamiento y el razonamiento mecánico, donde las diferencias son estadísticamente significativas. Con una fiabilidad del $95 \%$, no hay diferencias significativas asociadas al género en lo que respecta al factor cognitivo asociado al razonamiento mecánico.

Las iniciativas pedagógicas de la USI permiten profundizar en las actitudes y conductas de los estudiantes, que voluntariamente deciden participar (emergencias) y desde esa posición muestran su nivel de interés y compromiso con el conocimiento en física aplicada y matemáticas, mientras que, en las mujeres, aunque son constantes, muestran una considerable disminución en el interés por fortalecer sus capacidades en ciencia y tecnología asociadas a la discriminación previa.

\section{REFERENCIAS BIBLIOGRÁFICAS}

[1] Largo L, F, Rojas J, Vergel M 2020 La Empatía en el Aprendizaje de los estudiantes de educación básica desde la perspectiva de la interacción social. (Bogotá: Ecoe Ediciones)

[2] Carranza, R., Hernández, R., Alhuay, J. (2017) Bienestar psicológico y rendimiento académico en estudiantes de pregrado de psicología. Revista internacional de investigación en ciencias sociales 13, 2 .

[3] Santiago, M., Gallardo, H., Vergel, M. (2020) Resistencia en estudiantes exitosos en matemáticas. Revista Praxis\&Saber 11, 26.

[4] Vergel, M., Martínez, J., Nieto, J.F. (2016). Validez del instrumento de medición del aprendizaje creativo Revista Comunicaciones en Estadística 9, 2.

[5] Trejo, M., Llaven, G., Pérez, H. (2015). El enfoque de género en la educación. Revista Atenas 4, 32.

[6] Maccoby, E., Jacklin, C. (1974). The psychology of sex differences. Stanford: Stanford University Press.

[7] Radovic, S. (2018). Diferencias de género en el desempeño matemático en Chile. Revista Colombiana de Educación 74, 221. 
[8] Vergel Ortega, M, parra Lopez H y Martinez J. 2013 Metodología para elaborar planes de capacitación en instituciones de Educación superior - Revista logos, ciencia \& tecnología, 2013 MV Ortega, HMP López - Revista logos, ciencia \& tecnología, 2013Londres: Banco Mundial.

[9] Beilock, S., Gunderson, E., Ramírez, G., Levine, S. (2010) La ansiedad de las profesoras de matemáticas afecta el rendimiento de las niñas en matemáticas. PNAS 107, 5.

[10] Aronson, E., Wilson, T., Akert, R. (2010). Psicología Social. New Jersey: Upper Saddle River.

[11] Rojas, M., Correa, D. (2014). ¿Género en matemáticas? Un análisis de los resultados de las Olimpiadas matemáticas. Escenarios 12, 1.

12] Farfán, R. (2012). Socio-epistemología y ciencia. El caso del estado estacionario y su matemática. Barcelona: Gedisa.

[13] Madero, I. (2011) Inclusión y exclusión de género y clase dentro de la escuela chilena en 4 comunas del sur de Chile. Estudios pedagógicos 2, 37 .

[14] Mizala, A., Martínez, F., Martínez, S. (2015). Expectativas de los maestros de primaria sobre el rendimiento de los estudiantes: Cómo sus creencias se ven afectadas por su ansiedad por las matemáticas y el género de los estudiantes. Enseñanza y formación del profesorado 50, 7.

[15] Domínguez, P. (2002) Dando a la Mujer y a la Sociedad. Revista FAISCA de Alta Capacidad 9,1.

16] Alonso, G., Cantoral, R., Farfán, R., Marín, L., Méndez, C., Jaso, G., Robles, I., Vidal, R. (2013). Construcción social de la ciencia entre los niños en el Programa Niñdss Talento. México: Gobierno del Distrito Federal.

[17] Canché, E. (2013) Matemática educativa y equidad: un estudio socioepistemológico del talento en matemáticas. México: Centro de Investigación y de Estudios Avanzados del Instituto Politécnico Nacional.

18] Pita, S., Pértegas, S. (2002). Unidad de Epidemiología Clínica y Bioestadística. Complejo Hospitalario-Universitario Juan Canalejo. Cad Aten Primaria 9, 76.

[19] Zepeda, S., Abascal, R., López, E. (2015). Emociones: Factor de cambio en el aprendizaje. Revista Ra Ximhai 11, 4.

[20] Pérez-Jiménez, J. (2016). Educación con perspectiva de género en matemáticas. Hacia la inclusión y la relacionalidad en la era postmoderna. Revista Latinoamericana de Ciencias Sociales, Niñez y Juventud 14, 1.

21] Ursini, S., Ramírez, A., Mercado, M. (2017) Equidad, género y matemáticas en las escuelas mexicanas. Revista Colombiana de Educación 73. 